\title{
Pancreatic neuroendocrine carcinoma metastasis to the skin
}

\author{
Katarzyna Kuśnierz ${ }^{\circledR * 1}$, Anna Zemczak ${ }^{\circledR}{ }^{* 2}$, Bartosz Molasy33, Weronika Szczęsny-Karczewska ${ }^{\circledR 4}$, \\ Joanna Pilch-Kowalczyk ${ }^{5}$ \\ ${ }^{1}$ Department of Gastrointestinal Surgery, Medical University of Silesia, Katowice, Poland, \\ ${ }^{2}$ Department of Endocrinology and Neuroendocrine Tumours, Department of Pathophysiology and Endocrinology, \\ Medical University of Silesia, Katowice, Poland, \\ ${ }^{3}$ Students' Scientific Association of Medical University of Silesia, Department of Gastrointestinal Surgery, Katowice, Poland \\ ${ }^{4}$ Department of Pathomorphology and Molecular Diagnostics, Medical University of Silesia, Katowice, Poland \\ ${ }^{5}$ Department of Radiology and Nuclear Medicine, Medical University of Silesia, Katowice, Poland \\ * contributed equally
}

Key words: pancreas; neuroendocrine carcinoma; skin metastasis; lymph nodes

Neuroendocrine carcinoma (NEC) rarely metastasizes to the skin, and hence very few cases of cutaneous metastases of pancreatic NEC have been reported [1,2]. The identification of the primary site in metastatic NEC can be quite challenging because enlarged lymph nodes tend to be the first and only symptom. The prognosis and survival of patients with NEC is poor [1,2], and so it has been recommended that physical examination as well as biochemical, functional, and imaging tests be performed every 3 months [3]. A thorough physical examination of the skin should also be performed to look for possible metastases. Because our patient's clinical course proved extremely aggressive, we would like to strongly emphasize that diagnostic imaging should be repeated immediately prior to qualifying the patient for surgery. Correct determination of disease stage supports treatment planning and helps avoid unintended qualification for surgery.

A 49-year-old female patient started the diagnosis due to enlargement of the right inguinal lymph nodes. A lymph node packet $(52 \times 28 \times 36 \mathrm{~mm})$ revealed on ultrasound was excised. Histopathology of the surgical specimen showed metastasis of small cell NEC G3. Immunophenotyping revealed the following: ChrA (+), SYN $(+), \operatorname{AE} 1 / 3(+)(\operatorname{dot})$, TTF-1 (-), and a Ki-67 index of $65 \%$. Despite examination by two independent centres, the location of the primary tumour (TxN1Mx) was not identified.

In order to search for the primary lesion, computed tomography (CT) of the abdominopelvic cavity was performed, visualizing a packet of lymph nodes $(48 \times 32 \mathrm{~mm})$ on the right side of the lesser pelvis, at the level of the external iliac vessels. In somatostatin receptors scintigraphy with ${ }^{99 \mathrm{~m}} \mathrm{Tc}$-Tektrotyd revealed overexpression of somatostatin receptors in the lymph nodes seen at CT. The primary focus remained undetected on gynaecological and laryngological consultations, thyroid and breast ultrasound, colonoscopy, and chest CT. CT scans obtained 3 months later showed an enlarged nodal packet. ${ }^{18} \mathrm{~F}$-FDG PET/CT demonstrated a metabolically active process only in the external right iliac lymph nodes (SUVmax. 6.5) (Fig. 1A, B). Neuroendocrine tumour markers were normal. ${ }^{68} \mathrm{Ga}$-DOTATATE PET-CT 2 months later showed increased expression of somatostatin receptors (SUVmax 10.5 and 17.4) but only in the external right iliac lymph node packets of $59 \times 37 \mathrm{~mm}$ and $66 \times 41 \mathrm{~mm}$ in size. The patient was qualified for nodal packet excision and for the search for the primary lesion. Because the packets proved unresectable during surgery, the patient received palliative chemotherapy (cisplatin and etoposide), which was continued for 5 months. Abdominopelvic CT scan performed after the 6th cycle of chemotherapy showed almost complete regression.

Six months later, a ${ }^{18} \mathrm{~F}-\mathrm{FDG}$ PET-CT scan showed metabolic progression of the nodal infiltration in the pelvic area $(67 \times 47 \times 116 \mathrm{~mm})$. Due to the progression of the disease, locoregional advancement, and post-chemotherapy polyneuropathy, the patient was qualified for palliative hypofractionated radiotherapy (a total dose of $30 \mathrm{~Gy}$ in 10 fractions). Abdominal CT performed 3 months later revealed a solid 22-mm tumour of the pancreatic body, classified as the primary lesion (Fig. 2), and a stable disease in the pelvic lymph nodes according 

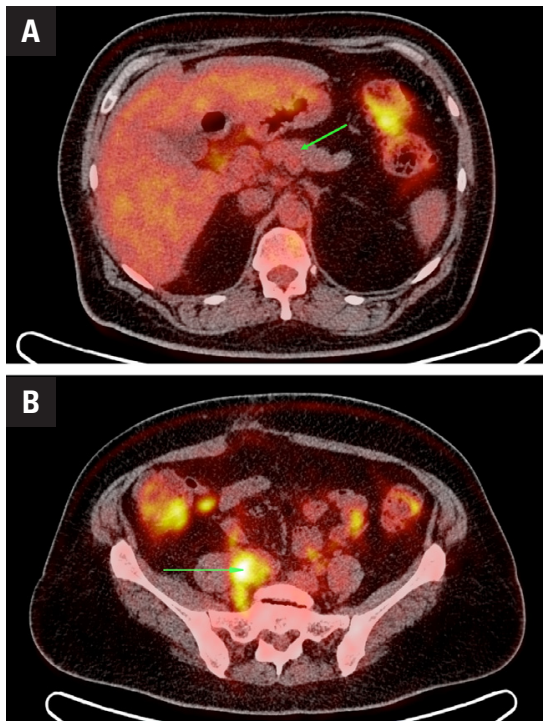

Figure 1. ${ }^{18} \mathrm{~F}-\mathrm{FDG}$ PET-CT. A. Normal pancreas (arrow). B. Metastatic lymph nodes on the right side of the lesser pelvis at the level of the external iliac vessels (arrow)

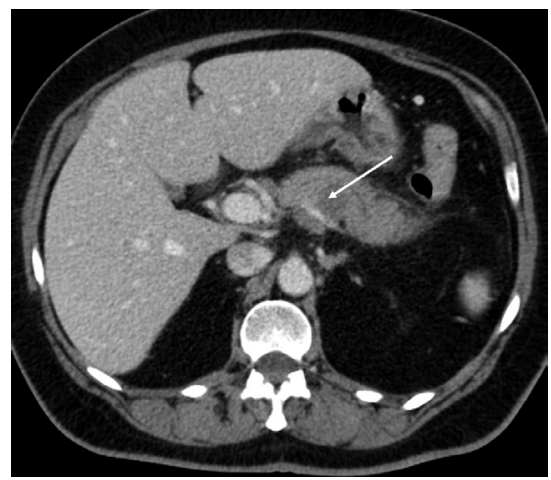

Figure 2. CT scan of the abdominopelvic cavity. Resectable tumour of the pancreatic body (arrow)

to Response Evaluation Criteria in Solid Tumours. The pancreatic tumour was considered resectable and the patient was qualified for elective surgery.

Two weeks after the above CT examination, the patient reported a rapidly enlarging tumour of the right forearm, now measuring $15 \times 30 \times 20 \mathrm{~mm}$. Another CT was performed prior to surgery for pancreatic tumour, revealing significant progression of the tumour to $31 \times 26 \mathrm{~mm}$, and metastatic lymph nodes $(51 \times 41 \times 87 \mathrm{~mm})$. Due to infiltration of the celiac trunk and hepatic artery (Fig. 3), the tumour was considered unresectable, and surgery was cancelled. Because the nature of the right forearm tumour had not been clarified, the lesion was excised. The histopathological examination revealed G3 NEC metastasis with a Ki-67 of $90 \%$ and mitotic index of 10/10 HPF (Fig. 4A, B). The patient was qualified for palliative oncological treat-

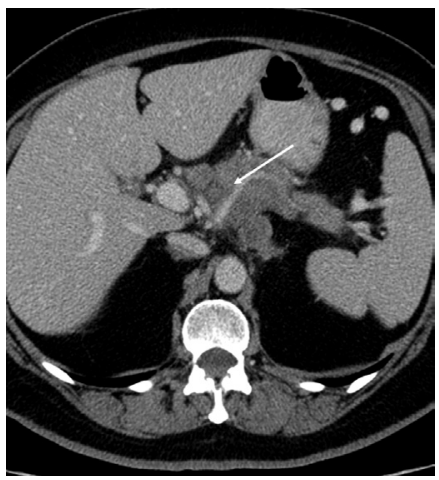

Figure 3. Abdominopelvic CT scan. Unresectable tumour of the pancreatic body with metastatic lymph nodes (arrow)

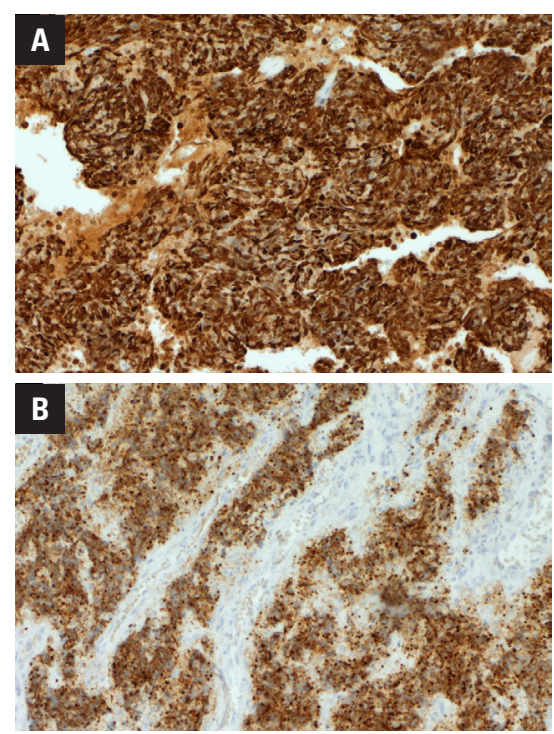

Figure 4. Histology with immunohistochemistry of NEC metastasis. A. Chromogranin A $(10 \times)$. B. Ki-67 index of approx. $90 \%(10 \times)$

ment, which she did not receive due to her rapidly deteriorating general condition. Unfortunately, the patient died 6 months later.

\section{Funding}

None declared.

\section{References}

1. Laschinger ME, Naga L, Gaspari AA. Cutaneous metastases of pancreatic neuroendocrine carcinoma. G Ital Dermatol Venereol. 2018; 153(5): 722-724, doi: 10.23736/S0392-0488.17.05103-3, indexed in Pubmed: 30246953.

2. Amorim GM, Quintella D, Cuzzi T, et al. Cutaneous Metastasis of Neuroendocrine Carcinoma with Unknown Primary Site: Case Report and Review of the Literature. Case Rep Dermatol. 2015; 7(3): 263-274, doi: 10.1159/000440661, indexed in Pubmed: 26557073.

3. Kos-Kudła B, Blicharz-Dorniak J, Strzelczyk J, et al. Consensus Conference, olish Network of Neuroendocrine Tumours. Pancreatic neuroendocrine neoplasms - management guidelines (recommended by the Polish Network of Neuroendocrine Tumours). Endokrynol Pol. 2013; 64(6): 459-479, doi: 10.5603/EP.2013.0031, indexed in Pubmed: 24431118. 